exposed to cardiopulmonary bypass (eg, no patient was supported with extracorporeal membrane oxygenation or a ventricular assist device).

As McConnell and Hibino point out, CorMatrix is a bioscaffold and the hope is that it will be remodeled and replaced by native tissue. That we saw minimal evidence for this was most concerning. Figure $1, B$, in the report of McConnell and Hibino extends these observations out past 1 year. We would strongly encourage clinicopathologic studies of explanted CorMatrix material from additional centers where the material is in clinical use for valve repair in congenital heart disease.

Stephen P. Sanders, MD

Robert F. Padera, Jr, MD, PhD

Boston Children's Hospital

Boston, Mass

http://dx.doi.org/10.1016/ j.jtcvs.2014.07.005

\section{INSUFFICIENCIES OF DOWNSIZING RING \\ ANNULOPLASTY IN \\ TREATMENT OF FUNCTIONAL ISCHEMIC MITRAL \\ REGURGITATION \\ To the Editor:}

The recent publication by Rabbah and colleagues ${ }^{1}$ directs attention to functional ischemic mitral regurgitation (MR; Figure 1, A) and underlying mechanisms that have not as yet been definitely addressed. The desired criterion standard surgical treatment has not yet been documented, and this issue requires further research. We appreciate Rabbah and colleagues ${ }^{1}$ for their efforts and would like to make some contributions.

The downsizing mitral annuloplasty (DSMA; Figure 1, $B$ ) is a surgical technique preferred by many surgeons that is effective in correcting annular dilatation. It is not effective, however, for left ventricular (LV) equatorial dilatations. We would like to discuss the DSMA, because it may be a reason for the poor long-term outcomes. This hypothesis has been supported by Magne and associates, ${ }^{2}$ who advocate that persistence of MR early after DSMA is related to the increased posterior leaflet tethering potentially caused by prosthetic ring implantation, which anteriorly displaces the posterior leaflet and reduces its mobility. From a different point of view, we think that there are two mechanisms causing poor outcomes. The first and initial deleterious effect of DSMA is in the isovolumetric contraction phase (IVCP) of the cardiac cycle, when both aortic and mitral valves are in the closed position (Figure 1, A). Ventricular pressure is sharply raised up to diastolic tension according to the volume-pressure relationship of the LV. In this period, the intraventricular volume is constant; DSMA, however, reduces the inflow cavity of the LV, which also results in further dilatation in the equatorial axis (Figure 1, $B$ ). The dilatation further increases the tethering forces on the chordae of mitral leaflet because there is no ejection in the IVCP yet. This is the triggering vicious circle, which continues until the surgically created coaptation line is broken down. Once the coaptation line has disappeared by means of spherical dilatation caused by DMSA in adverse ventricular remodeling, there is recurrent MR, and after this stage the second phase (never ending vicious circle) is engaged and gains autonomy. In each cardiac cycle, recurrent MR further causes LV dilatation, which in turn results in increased LV sphericity.

Rabbah and colleagues ${ }^{1}$ reported, "The large patch procedure with true-size annuloplasty resulted in the best improvement in coaptation length, and decreased tethering forces on the chords of posterior papillary muscle when compared with the functional ischemic MR group." In another study by Magne and associates, ${ }^{3}$ it was demonstrated in a "nonpersistent MR group" that the coaptation depth was smaller than that in a "persistent MR group" (8.1 $\pm 2 \mathrm{~mm}$ vs $11.7 \pm 2 \mathrm{~mm} ; P<.01)$. Moreover, the angle between the annular plane and leaflets was smaller than in the persistent MR group ( $36^{\circ}$ $\pm 7.5^{\circ}$ vs $\left.53^{\circ} \pm 6^{\circ} ; P<.01\right)$. In other words, deeper coaptation depth and larger angle in the persistent MR group mean more downsizing, which explains the greater tethering in the IVCP. This result proves that the increased tethering in IVCP is the result of increased equatorial axis of the LV.

We believe that the never ending vicious circle can be broken by breaking the triggering vicious cycle with true sizing and patch augmentation, which may result in better outcomes. This point of view may be helpful for surgeons to improve the poor outcomes of functional ischemic MR in longterm follow-up.

Murat Tavlasoglu, $M D^{a}$ Mustafa Kurkluoglu, $M D^{b}$ Ahmet Baris Durukan Adem Guler ${ }^{b}$

${ }^{a}$ Department of Cardiovascular Surgery

Diyarbakir Military Medical Hospital Diyarbakır, Turkey

${ }^{b}$ Department of Cardiovascular Surgery

Gulhane Military Medical Hospital Ankara, Turkey

${ }^{c}$ Department of Cardiovascular Surgery

Memorial Hospital Ankara, Turkey

\section{References}

1. Rabbah JP, Siefert AW, Bolling SF, Yoganathan AP. Mitral valve annuloplasty and anterior leaflet augmentation for functional ischemic mitral regurgitation: quantitative comparison of coaptation and subvalvular tethering. Journal Thorac Cardiovasc Surg. April 13, 2014 [Epub ahead of print].

2. Magne J, Pibarot P, Dumesnil JG, Sénéchal M. Continued global left ventricular remodeling is not the sole mechanism responsible for the late recurrence of ischemic mitral regurgitation after restrictive annuloplasty. J Am Soc Echocardiogr. 2009;22:1256-64

3. Magne J, Pibarot P, Dagenais F, Hachicha Z, Dumesnil JG, Sénéchal M. Preoperative posterior 


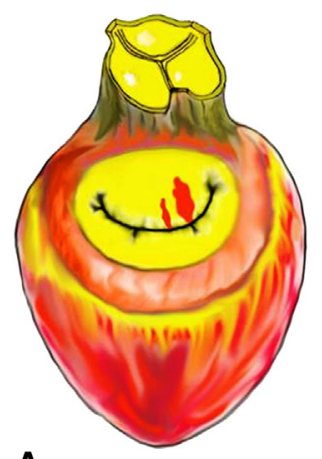

A

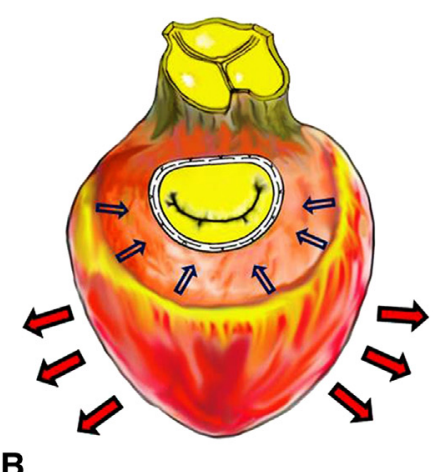

B

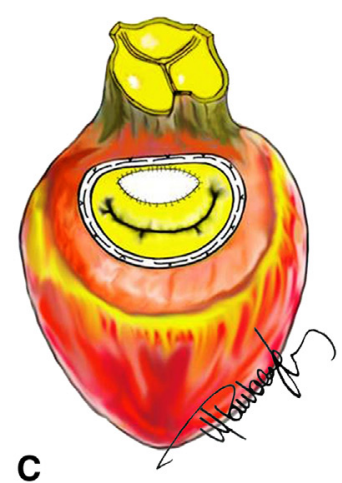

FIGURE 1. The illustration demonstrates the isovolumetric contraction phase of the left ventricle. Note that both aortic and mitral valves are in the closed position, and the atria and right ventricle are removed. A, The illustration demonstrates the occurrence of mitral regurgitation in functional ischemic mitral regurgitation. B, The illustration demonstrates the annular downsizing, equatorial dilatations, and increased sphericity that may be the deleterious effects of downsizing mitral annuloplasty during the isovolumetric contraction phase. C, The illustration demonstrates true sizing mitral annuloplasty and patch augmentation.

leaflet angle accurately predicts outcome after restrictive mitral valve annuloplasty for ischemic mitral regurgitation. Circulation. 2007;115:782-91.

\section{http://dx.doi.org/10.1016/} j.jtcvs.2014.06.034

\section{Reply to the Editor:}

Advances in mitral valve repair have taught us that judicious annular downsizing with a complete rigid ring abolishes ischemic mitral regurgitation (IMR) in most cases. In a select subgroup, however, downsized annuloplasty can fail, resulting in persistent or recurrent mitral regurgitation. In analyzing these patients, preoperative predictors of repair failure have been advocated that may potentially aid future procedure selection. ${ }^{1}$ Despite this knowledge, uncertainty remains in selecting the best procedure for eliminating mitral regurgitation.

The importance of surgically eliminating mitral regurgitation was highlighted in a recent clinical trial assessing the relative effectiveness of annuloplasty and replacement in severe IMR. ${ }^{2}$ This study reported no significant differences at 12 months in reverse ventricular remodeling, survival, and clinical outcomes between patients undergoing mitral valve annuloplasty and replacement. Conclusions drawn from this study, however were dampened by the finding that $32 \%$ of patients undergoing annuloplasty exhibited significant recurrent mitral regurgitation, reduced left ventricular remodeling, and impaired survival. This patient subset emphasizes the importance of selecting repairs that terminate IMR and prohibit persistent or recurrent regurgitation. Moreover, it is necessary to categorize future IMR outcomes into 3 distinct groups: repair, replacement, and repair with persistent or significant recurrent regurgitation.

The letter by Tavlasoglu and colleagues further underlines the importance of improving patient outcomes by selecting repairs that eliminate IMR. In cases in which downsized annuloplasty is anticipated to be suboptimal, we agree that adjunct techniques may assist in maximizing coaptation length, reducing tethering, and ceasing regurgitation. As described by Tavlasoglu and colleagues, this is particularly critical when extensive equatorial left ventricular dilatation and chordalization of the posterior leaflet is observed. Beyond leaflet augmentation, other adjunct techniques have demonstrated success and should be considered. These include papillary muscle relocation, papillary muscle approximation, chordal cutting, and external ventricular constraint. ${ }^{3,4}$ Regardless of the chosen technique, 2 fundamental goals are consistent in these procedures: maximizing leaflet coaptation and reducing subvalvular tethering. Only when elimination of IMR cannot predictably be achieved by repair should selective replacement be considered.

In light of current controversies in patient and procedure selection, a critical need exists to evaluate IMR surgical techniques on the basis of robust quantitative data. Clinical, animal, and in vitro studies must continue to address this challenge directly by providing evidence-based insight into procedure selection in targeted subsets of patients with IMR. $^{2-4}$ Clinical decisions made from robust quantitative data will improve methods for eliminating IMR and potentially improve longterm patient outcomes. These efforts are important now more than ever to address and treat the significant and growing clinical burden of IMR.

Andrew W. Siefert, $P h D^{a, b}$ Jean-Pierre M. Rabbah, $P h D^{b}$

Steven F. Bolling, $M D^{c}$

Ajit P. Yoganathan, $P h D^{b}$

${ }^{a}$ Exponent Engineering and Scientific Consulting

Philadelphia, $\mathrm{Pa}$

${ }^{b}$ Wallace H. Coulter Department of Biomedical Engineering 